Article

\title{
Fluctuating Asymmetry in Flies, What Does It Mean?
}

\author{
Athol J. McLachlan \\ Biology Department, University of Newcastle upon Tyne, NE1 7RU, UK; \\ E-Mail: a.j.mclachlan@virgin.net
}

Received: 23 February 2010; in revised form: 11 May 2010 / Accepted: 27 May 2010 /

Published: 4 June 2010

\begin{abstract}
The degree of departure from perfect symmetry in organisms, fluctuating asymmetry (FA), is seen in most populations of animals. It has particular impact on choice of mate which lies within the world of sexual selection. Here I consider a relatively little studied aspect of sexual selection, i.e. the effect of FA on contests between males for mates, based not on display ornament but rather on agility seen in the mating systems of many insects. The model organism considered is the ubiquitous chironomid midge. In these flies, mating takes place in the air, so symmetry in the length of wings bears directly on a male's aerobatic ability on which successful mating depends. The role of parasites and predators in creating and responding to FA in the host/prey midge is considered.
\end{abstract}

Keywords: biomechanics; chironomid midges; fluctuating asymmetry; mating flight; parasites; predators; sexual selection

\section{Introduction}

At least since the work of Isaac Newton (1642-1727), physicists and chemists have grappled with universal symmetry and its implications for understanding the nature of the universe [1]. It is only recently that biologists have become interested in symmetry per se and in departures from perfect symmetry too [2]. The subject has become a matter of intense interest and research and several different kinds of symmetry breaking have been recognized in animals. These include directional asymmetry present, for example, in many of the internal organs of mammals and the shells of snails, antisymmetry seen in the chelae (claws) of many crabs, and bilateral symmetry in the external appearance (morphology) of man, flies and many others [3]. It is the last of these that I wish to consider here. Within populations of animals with an essentially bilaterally symmetrical body plan, minute deviations to the left or right are know as fluctuating asymmetry (FA) [4]. 
For many years the evident near perfection in the morphology of animals, seemingly beyond that required by natural selection, has engaged my curiosity but has been in want of an explanation. So, the mention of FA over lunch with Matt Ridley, some years ago, immediately attracted my attention. Charles Darwin was struck by the same point over 150 years before me and wrote "beauty is apparently wholly due to symmetry of growth" [5]. Not surprisingly therefore, an aspect of FA that prompted early interest was the striking symmetry between the left and right sides of the human face. There is evidence that the degree of symmetry here is readily detected by potential mates [6-8], which places studies of FA firmly in Darwin's sexual selection realm. Departure from perfect symmetry is taken to be a measure of instability during development called developmental instability (DI). By this is meant that the more closely perfect symmetry is approached, the more robust the organism in the face of environmental or genetical stress during development [9].

Observations of such different animals as birds and insects have shown that the degree of symmetry in sexual display ornaments such as the tails of swallows, and the horns of beetles is important in choice of mate [10]. In this world of sexual selection, the general rule is that females choose males on the basis of the quality of their sexual ornaments and since this in turn reflects the level of DI. FA in sexual ornament is thought to be a reliable physical (phenotypic) measure of genetic quality. As a consequence, in accepting a male with high levels of symmetry in his ornaments, a female gains genetical benefits for her offspring. It is for these reasons that the evolution of ornament based choice is favoured by sexual selection [11]. Hence, FA is widely used as a measure of fitness. The value of such a readily measurable indicator of fitness will not be lost on a biologist. To quote Joseph Tomkins and Leigh Simmons, "rarely in biology has there been such an intuitively attractive and broadly applicable proposition" [9].

Here I take a single taxon with which I am closely familiar-the ubiquitous chironomid midge, and use this as a model organism with which to examine FA and its consequences for mate choice and mating success. I go further and consider how the study of predators and parasites of the midge provided insights into FA in the functioning of an elusive and fascinating mating system. Chironomids have adopted a swarm based mating system, reviewed by [12], and thus the biomechanics of mating on the wing lies at the centre of their mating behavior [13]. This contrast with ornament based mating systems which have provided the main focus of research on FA. My hope in choosing to consider the mating system of chironomids is to bring attention to bear on a widespread but less studied aspect of sexual selection.

\section{FA in Chironomid Midges}

\subsection{Some Background on the Biology of the Chironomid Midge}

Chironomids are holometabolous insects, which mean that once they emerge as adults there is no further growth. This has profound consequences for their biology and for the study of their mating behaviour. For example, as adults these midges do not need to feed at all [14]-in short they have a mayfly type of life-cycle. Here the adult functions solely as a mating and reproducing stage which greatly simplifies the testing of hypotheses concerning adaptation within the mating system. Further simplification is due to the brief life-span typical of may-fly like animals. I have suggested that, given 
this life-cycle constraint, the likelihood is that any particular male midge would have only a single evening in which to achieve a mating. This is in marked contrast to many other insects, including dragonflies and damselflies, and results in very different selection pressures on the mating system. Larger insects such as the latter two have received significantly more attention than the tiny midges. Biologists working with larger insects appear not readily to perceive this life span related difference in the mating system [15].

Once metamorphosed as adults, male midges typically aggregate in aerial swarms numbering thousands of individuals (Figure 1) [12].

Figure 1. A mating swarm of male chironomid midges above trees on a windless evening.

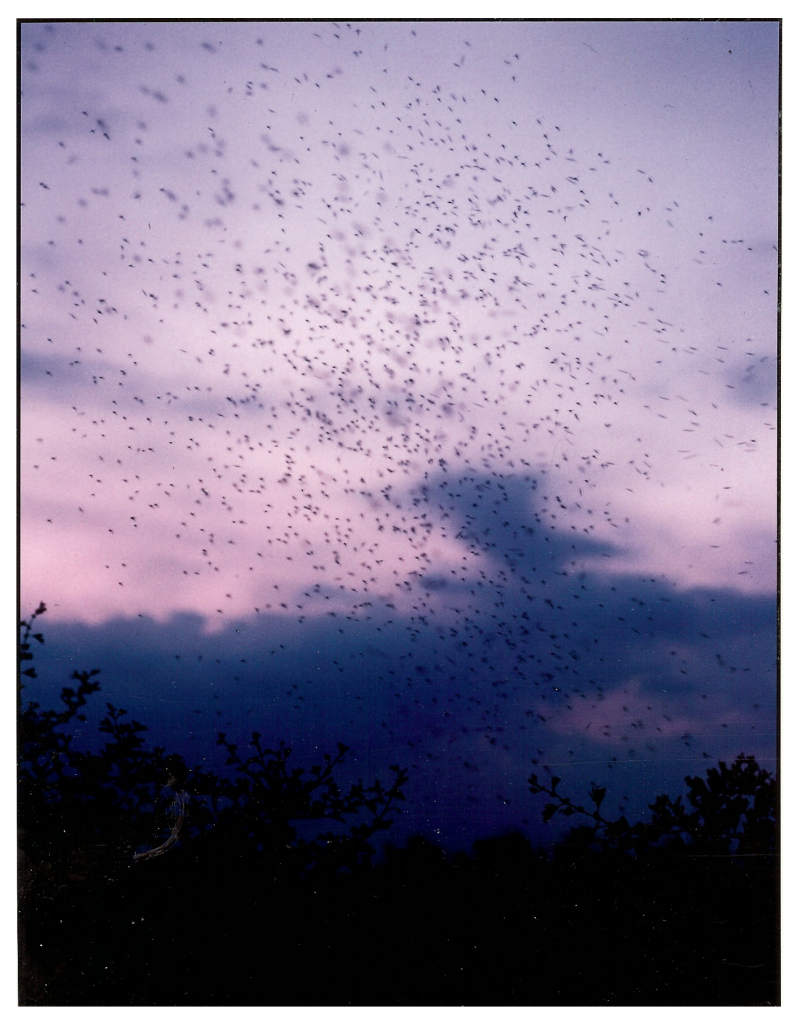

Females seek the swarms which they enter to emerge in tandem with a male. The fertilized female then departs on an egg laying flight. In such an arrangement, sexual selection appears to favor aerobatic ability in the male [13], where access to females may involve competition for a favorable location within the mating swarm [16]. Hence anything that perturbs aerobatic performance tends to reduce mating success and hence male fitness. It is for this reason that asymmetries in the wings of adults, measured as FA, and their consequences for flight performance, are a key to understanding such a mating system.

\subsection{Biomechanics of Mating Flight in Midges}

Much of the work on perturbations of flight due to wing asymmetry in midges has been confounded by the effects of body size which is inversely related to flight performance [17], and therefore also to mating success. As a consequence, midges gaining in the mating stakes tend to be both the smallest and the most symmetrical. In an attempt to separate FA and size, measurements of both variables were 
made in common midge species, both from swarms and from mating pairs. The assumption here is that males in mating pairs will be successful at passing on their sperm while those in the swarm are the unsuccessful part of the male population. The assumptions and precautions behind this approach are discussed elsewhere $[18,19]$. Results suggest that it could be the degree of symmetry associated with small size, rather than size per se, that accounts for the success of the small males [20]. It has been proposed that this result is due to the aerodynamic effects of wing symmetry on flight performance; an hypothesis supported by an experiment where asymmetry was increased artificially by adding droplets of aerosol paint to swarms in the wild. Untreated swarms provide a control treatment [21]. This approach, involves that most powerful tool in the suite of scientific methods-the experiment. It is promising but difficult in that context and requires refinement.

\subsection{Parasites, Predators and FA in Midges}

Parasites as a force shaping adaptive change in animals had been largely overlooked until the ground breaking work of Bill Hamilton and colleagues [22-24]. Here I attempt two things concerning infection by parasites. First, I present evidence to show that infection by a parasite can increase the asymmetry of the infected host individual. Second, I will attempt to show that both parasites and predators appear to use asymmetry in the host midge in discriminating between individuals as suitable hosts.

Evidence for the first of these is widespread. Studies in many animals, notably birds and insects, demonstrate that infection produces stress during development so that infected individuals tend to express high levels of DI and hence also high levels of asymmetry [25,26]. The consequence is impaired mating success and fitness. Among midges, the phenotypic effects of parasite induced asymmetry on mating success have several layers. These include favored spatial location in a mating swarm [16], and biomechanical perturbations on flight performance due to wing asymmetry [20,21]. But where ectoparasites are present there is another effect-the physical location of an ectoparasitic on the body of the host. Infection by ectoparasitic mites is common among insects where the parasite is typically large relative to the host (Figure 2). Parasites can therefore be predicted to cause perturbations in flight due to load and drag affects [27].

Because males appear to depend largely on aerobatics to achieve a mating, an asymmetrical placement of mites would be predicted to be damaging to the mating chances of the host male. The survival of the parasite too, may be influenced by placement on the host. For example, the female midge departs on an egg laying flight only after she has mated, reviewed by [28]. Since mites depend on the female midge for transport to water where they complete their life-cycle, there may be fitness benefits for mites to arrange symmetrically on the host so as not to interfere with host mating behaviour [27]. These predictions are entirely born out by the evidence. The degree of symmetry in the arrangement of mites, the Symmetry Quotient (SQ), is a kind of second level FA which may be a response to the primary asymmetry in the host or to anti-parasite behaviour in the host, such as grooming [27]. Neither the putative effect of FA on SQ nor host anti-parasite behaviour has been formally investigated. 
Figure 2. A male chironomid midge bearing three mites, two of which are asymmetrically implanted. Scale line; $1 \mathrm{~mm}$.

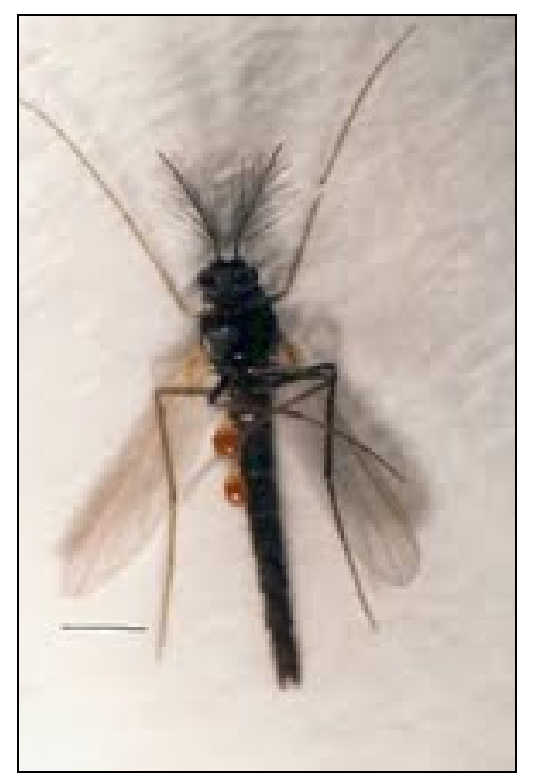

The second thing I wish to consider concerning the effect of parasites is the effect of asymmetry on choice by the parasite. Mites select host individual in the aquatic pupal stage and stay with them until they emerges as adults, at which time mites implant [29]. So any preference for host based on asymmetry, or its correlates, must be sought by the mite in the pupal stage. Findings suggest that mites tend to associate with the most asymmetrical pupae and therefore carry through to the most asymmetrical adult. There are many consequences which differ in detail in the male and female midge, fully discussed by McLachlan and co-authors [27]. One such is that adult male midges, but not female, experience enhanced turn-rate in flight when carrying an asymmetrical load. The possibility is that flight instability imparted by the asymmetrical load involves an aspect of aerobatics that impacts on both mating success and predator avoidance in the male. An analogy to aircraft is appropriate; built-in instability in flight correlates in fighter air craft with rapid aerobatic response, particularly turn rate, which is an effective maneuver in avoiding attack [30]. Following this line of reasoning, there could be fitness benefits for the mite on a male midge with high SQ levels. But, asymmetry induced by the presence of the mite could also impair the mating chances of a male midge. Furthermore this could have undesirable consequences for the mite on a female midge because females will only depart to water after mating. Thus the mite on an unmated female midge will be unable to complete its life-cycle and hence have zero fitness. Implications of the finding that parasites may actively choose their host on the basis of FA has far reaching consequences for predictions of individual probability of parasite induced disease in animals including man [31].

Predators are a well known force shaping adaptation and FA in prey animals [32-35]. The predators of midges show some close parallels to the effect of parasites. For example, any asymmetry in wing length might be expected to increase the chance of predation through impaired aerobatics in the prey. This prediction has been tested on male midge swarms which are routinely predated by male dace flies (empids) (Figure 3), searching for male midges as nuptial gifts for their own females (Mclachlan, A. J. and Ladle, R., unpublished data). 
Figure 3. A male empid fly (top) is providing a nuptial gift of a midge (bottom) to a females empid (middle). (Photo by Funk, D. H., Stroud Water Research Center, Avondale, PA, USA. Reproduced with permission of the Royal Society, front cover, Phil. Trans, 2006, 361).

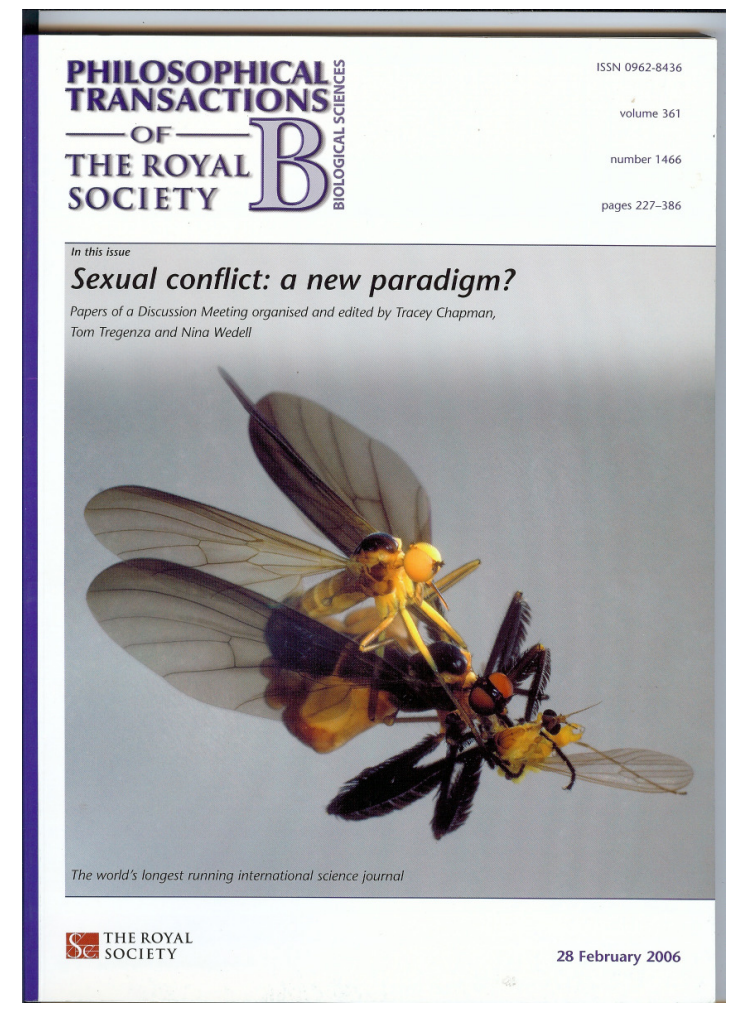

These predacious flies enter the midge swarm and emerge clasping a male midge. As expected, the more asymmetrical male midges are preferentially captured. The effect of an asymmetrical placement of mites on the body of the host would be interesting in this context but was not followed up. As expected though, the most symmetrical empids are most successful at capturing chironomids.

\section{Conclusions}

I here draw out the two aspect of FA in sexual selection that would benefit from some emphasis. The theme that runs through this review is that mating success within many mating systems may depend, not on quality of sexual display, but rather on biomechanical proficiency which in turn hinges of FA. The example I draw attention to is the common chironomid midge, which mates on the wing in aerial mating swarms seen also in many other insect species [12]. Aerobatic ability lies at the core of such mating systems that operate in a three dimensional medium, seen also in many species of fish and animals mating in an arboreal space, such as simians [36]. I exclude work on FA in bird flight aerodynamics e.g. [37], because, as far as I am aware and with the possible exception of swifts, birds do not mate on the wing. The findings I present have wide application to understanding an aspect of sexual selection where FA affects the biomechanics of mating. This largely neglected type of mating system will reward attention, particularly because technology, in the form of high-speed video recording equipment, offers a tool for close observation of acrobatics in the wild [13,38]. The emphasis on the biomechanical consequences of FA in an aerial mating system is not meant to exclude 
other possible effects such as direct visual mechanisms, which are known to be important in the biology of some insects [39].

I point up a second largely overlooked aspect of FA, that is its effect on the susceptibly to parasites in host animals. The implication of this finding may prove applicable to a wide range of parasites and host species including man. I refer to the possibility that it may be possible to predict which individuals within a population are vulnerable to infection as a consequence of phenotypic asymmetry. This possibility feds into current attempts to target medical treatment based on individual peculiarities which may be reflected in the individual's level of symmetry which in turn reflects individual genomic characteristics, reviewed by $[40,41]$.

It has not escaped my attention that there must be strong selective forces at work on FA between the host and its attempts to mate and reproduce on the one hand and FA in its parasites and predators on the other. Thus FA is at the centre of a potential evolutionary arms race of the kind beautifully encapsulated in the Red Queen Hypotheses [42].

Some caution is required in interpreting the fitness consequences of FA. There are several reasons for reaching this conclusion but prominent among them is the characteristically small size of departures from prefect symmetry in phenotypic characters. This property of FA leads to both measurement and statistical difficulties. I do not dwell on these but the whole matter is masterfully and comprehensively explored by Polak and colleagues [43], to whom the reader is directed. The same authors show that the study of FA lays a solid foundation for a burgeoning new field of endeavor in evolutionary biology.

\section{Acknowledgements}

The comments of two unnamed reviewers are acknowledged.

\section{References and Notes}

1. Icke, V. The Force of Symmetry. Nature 1995, 276, 32.

2. Ludwig, W. Das Rechts-links Problem im Tierreich und Beim Menschen; Springer: Berlin, Germany, 1932.

3. Klingenberg, C.P.A. Developmental perspective on developmental instability: Theory, models, and mechanisms. In Developmental Instability: Causes and Consequences; Polak, M., Ed.; Oxford University Press: Oxford, UK, 2003.

4. Van Valen, L. A study of fluctuating asymmetry. Evolution 1962, 16, 125-142.

5. Darwin, C. The Origin of Species by Means of Natural Selection, or the Preservation of Favoured Races in the Struggle of Life; John Murray: London, UK, 1859.

6. Enquist, M.; Arak, A. Symmetry, beauty and evolution. Nature 1994, 372, 169-172.

7. Gangestad, S.W.; Thornhill, R.; Yeo, R.A. Facial attractiveness, developmental stability and fluctuating asymmetry. Ethol. Sociobiol. 1994, 15, 73-85.

8. Grammer, K.; Thornhill, R. Human (Homo sapiens) facial attractiveness and sexual selection: The role of symmetry and averageness. J. Comp. Psychol. 1994, 108, 233-242. 
9. Tomkins, J.L.; Simmons, L.W. Fluctuating asymmetry and sexual selection: Paradigm shifts, publication bias, and observer expectation. In Developmental Instability: Causes and Consequences; Polak, M., Ed.; Oxford University Press: Oxford, UK, 2003; pp. 231-261.

10. Møller, A.P.; Cuervo, J.J. Asymmetry, size, and sexual selection: Factors affecting heterogeneity in relationships between asymmetrty and sexual selection. In Developmental Instability: Causes and Consequences; Polak, M., Ed.; Oxford University Press: Oxford, UK, 2003, pp. 262-278.

11. Andersson, M. Sexual Selection; Princeton University Press: Princeton, NJ, USA, 1994.

12 McLachlan, A.J.; Neems, R.M. Swarm based mating systems. In Insect Reproduction; Leather, S.R., Hardie, J., Eds.; CRC Press: New York, NY, USA, 1995.

13. Crompton, B.; Thomason, J.; McLachlan, A.J. Mating in a viscous universe: the race is to the agile, not to the swift. Proc. Roy. Soc. London Ser. B 2003, 270, 1991-1995.

14. Burtt, E.T.; Perry, R.J.; McLachlan, A.J. Feeding and sexual dimorphism in adult midges. Holarctic Ecol. 1986, 9, 27-32.

15. McLachlan, A.J. Male mating success in diptera: A reply to Thompson. Oikos 1988, 51, 109.

16. Pike, T.W. Fitness effects of parasite-mediated spatial heterogeneity within a swarm. Behav. Ecol. 2006, 17, 992-997.

17. Neems, R.M.; Lazarus, J.; McLachlan, A.J. Lifetime reproductive success in a swarming midge: Trade-offs and stabilizing selection for male body size. Behav. Ecol. 1998, 9, 279-286.

18. McLachlan, A.J.; Neems, R.M. An alternative mating system in small male insects. Ecol. Entomol. 1989, 14, 85-91.

19. McLachlan, A. Parasites promote mating success: The case of a midge and a mite. Anim. Behav. 1999, 57, 1199-1205.

20. McLachlan, A.J.; Cant, M. Small males are more symmetrical: Mating success in the midge Chironomus plumosus L. (Diptera: Chironomidae). Anim. Behav. 1995, 50, 841-846.

21. McLachlan, A.J. Size or symmetry: An experiment to determine which of the two accounts for mating success in male midges. Ecoscience 1996, 4, 454-459.

22. Emlen, S.T. Introduction to William D. (Hamilton symposium). Behav. Ecol. 2001, 12, 261.

23. Milinski, M. Bill Hamilton, sexual selection and parasites. Behav. Ecol. 2001, 12, 24-26.

24. Schmid-Hempel, P. Wondering about sex: W. K. Hamilton's contribution to explaining nature's masterpiece. Behav. Ecol. 2001, 12, 266-268.

25. Polak, M.; Møller, A.P.; Gangstad, S.W.; Kroeger, D.E.; Manning, J.T.; Thropnhill, R. Does an individual asymmetry parameter exist? In Developmental Instability: Causes and Consequences; Polak, M., Ed.; Oxford University Press: Oxford, UK, 2003; pp. 81-96.

26. Møller, A.P. A Review of Developmental Instability, Parasitism and Disease: Infection, Genetics and Evolution; Elsevier: London, UK, 2006.

27. McLachlan, A.J.; Pike, T.W.; Thomason, J.C. Another kind of symmetry: Are there adaptive benefits to the arrangement of mites on an insect host? Ethol. Ecol. Evol. 2008, 20, 257-270.

28. Downes, J.A. The swarming and mating flight of diptera. Annu. Rev. Entomol. 1969, 14, 171-297.

29. McLachlan, A.J.; Ladle, R.; Bleay, C. Is infestation the result of adaptive choice behaviour by the parasite? A study of mites and midges. Anim. Behav. 1999, 58, 615-620.

30. Doyle, A. Better control for aircraft instability. Flight Int. 1995, 148, 34-36. 
31. Hart, B.L. Behavioral adaptations to pathogens and parasites: Five strategies. Neurosci. Biobehav. Rev. 1990, 14, 273-294.

32. Møller, A.P. Differential predation costs of a secondary sexual character: Sparrow hawk predation on barn swallows. Anim. Behav. 1997, 54, 1545-1551.

33. Møller, A.P. Sexual selection, viability selection, and developmental stability in the domestic fly Musca domestica. Evolution 1996, 50, 746-752.

34. Swaddle, J.P. Developmental stability and predation success in an insect predator prey system. Behav. Ecol. 1997, 8, 433-436.

35. Sneddon, L.U.; Swaddle, J.P. Asymmetry and fighting performance in the shore crab Carcinus maenas. Anim. Behav. 1999, 58, 431-435.

36. Alexander, R.D.; Hoogland, R.D.; Noonan, K.M.; Sherman, P.W. Sexual selection dimorphisms and breeding systems in pinnipeds, ungulates, primates and humans. In Evolutionary Biology and Human Behaviour; Charton, N.A., Irons, W.G., Eds.; Duxbury Press: North Scituate, MA, USA, 1978; pp. 402-435.

37. Thomas, A.L.R. The aerodynamic costs of asymmetry in the wings and tail of birds: Asymmetric birds can't fly round tight corners. Proc. Roy. Soc. London Ser. B 1993, 254, 181-189.

38. Buchen, L. Flies on film. Nature 2009, 462, 562-564.

39. Giufa, M.; Eichmann, B.; Menzel, R. Symmetry perception in an insect. Nature 1996, 382, $458-461$.

40. Collins, F.S. The language of life: DNA and the revolution in personalized medicine; Harper: London, UK, 2010.

41. Thornhill, R.; Møller, A.P. Developmental stability, disease and medicine. Biol. Rev. 1997, 72, 497-548.

42. Van Valen, L. A new evolutionary law. Evol. Theor. 1973, 1, 1-30.

43. Polak, M. Developmental Instability: Causes and Consequences; Oxford University Press: Oxford, UK, 2003.

(C) 2010 by the authors; licensee MDPI, Basel, Switzerland. This article is an Open Access article distributed under the terms and conditions of the Creative Commons Attribution license (http://creativecommons.org/licenses/by/3.0/). 